\title{
A CLASS OF COMPLETELY MONOTONIC FUNCTIONS INVOLVING DIVIDED DIFFERENCES OF THE PSI AND TRI-GAMMA FUNCTIONS AND SOME APPLICATIONS
}

\author{
Bai-Ni Guo And Feng QI
}

\begin{abstract}
A class of functions involving divided differences of the psi and tri-gamma functions and originating from Kershaw's double inequality are proved to be completely monotonic. As applications of these results, the monotonicity and convexity of a function involving the ratio of two gamma functions and originating from the establishment of the best upper and lower bounds in Kershaw's double inequality are derived, two sharp double inequalities involving ratios of double factorials are recovered, the probability integral or error function is estimated, a double inequality for ratio of the volumes of the unit balls in $\mathbb{R}^{n-1}$ and $\mathbb{R}^{n}$ respectively is deduced, and a symmetrical upper and lower bounds for the gamma function in terms of the psi function is generalized.
\end{abstract}

\section{Introduction}

Recall [28, Chapter XIII] and [48, Chapter IV] that a function $f$ is said to be completely monotonic on an interval $I$ if $f$ has derivatives of all orders on $I$ and

$$
(-1)^{n} f^{(n)}(x) \geq 0
$$

for $x \in I$ and $n \geq 0$. The famous Bernstein's Theorem in [48, p. 160, Theorem 12a] states that a function $f$ is completely monotonic on $[0, \infty)$ if and only if

$$
f(x)=\int_{0}^{\infty} e^{-x s} \mathrm{~d} \mu(s)
$$

Received March 23, 2010.

2010 Mathematics Subject Classification. 05A10, 26D15, 26A48, 26A51, 33B15, 33B20.

Key words and phrases. completely monotonic function, divided difference, gamma function, psi function, tri-gamma function, probability integral, error function, double factorial, ratio, volume of unit ball, monotonicity, convexity, inequality, generalization, application.

The second author was supported in part by the Science Foundation of Tianjin Polytechnic University. 
where $\mu$ is a nonnegative measure on $[0, \infty)$ such that the integral $(2)$ converges for all $x>0$. This expresses that a completely monotonic function $f$ on $[0, \infty)$ is a Laplace transform of the measure $\mu$.

It is well-known that the classical Euler's gamma function

$$
\Gamma(x)=\int_{0}^{\infty} t^{x-1} e^{-t} \mathrm{~d} t
$$

for $x>0$, its logarithmic derivative, denoted by $\psi(x)=\frac{\Gamma^{\prime}(x)}{\Gamma(x)}$, and the polygamma functions $\psi^{(i)}(x)$ for $i \in \mathbb{N}$ are several of the most important special functions and have much extensive applications in many branches such as statistics, probability, number theory, theory of 0-1 matrices, graph theory, combinatorics, physics, engineering, and other mathematical sciences.

The ratio $\frac{\Gamma(x+p)}{\Gamma(x+q)}$ for $x+p>0$ and $x+q>0$ of two gamma functions, called Wallis function or ratio in the literature, has been investigated at least since 1948 in [47]. Now there exist a lot of conclusions on Wallis ratio, its variants, generalizations and applications, for example, $[2,9,16,18,19,26,29,30,31$, $35,38,41]$ and related references therein.

In $[24]$, D. Kershaw proved a double inequality

$$
\left(x+\frac{s}{2}\right)^{1-s}<\frac{\Gamma(x+1)}{\Gamma(x+s)}<\left(x-\frac{1}{2}+\sqrt{s+\frac{1}{4}}\right)^{1-s}
$$

for $0<s<1$ and $x \geq 1$. It is clear that the inequality (4) can be rearranged as

$$
\frac{s}{2}<\left[\frac{\Gamma(x+1)}{\Gamma(x+s)}\right]^{1 /(1-s)}-x<\sqrt{s+\frac{1}{4}}-\frac{1}{2} .
$$

This suggests us to introduce a function

$$
z_{s, t}(x)= \begin{cases}{\left[\frac{\Gamma(x+t)}{\Gamma(x+s)}\right]^{1 /(t-s)}-x,} & s \neq t \\ e^{\psi(x+s)}-x, & s=t\end{cases}
$$

on $x \in(-\alpha, \infty)$ for real numbers $s$ and $t$ and $\alpha=\min \{s, t\}$.

In $[11,17,21,44]$, the monotonic and convex properties of $z_{s, t}(x)$ were established by using Laplace transform and other complicated techniques. Their basic calculation is as follows:

(8) $z_{s, t}^{\prime \prime}(x)=\left[z_{s, t}(x)+x\right]\left\{\left[\frac{\psi(x+t)-\psi(x+s)}{t-s}\right]^{2}+\frac{\psi^{\prime}(x+t)-\psi^{\prime}(x+s)}{t-s}\right\}$

$(9)=\frac{z_{s, t}(x)+x}{(t-s)^{2}}\left\{[\psi(x+t)-\psi(x+s)]^{2}+(t-s)\left[\psi^{\prime}(x+t)-\psi^{\prime}(x+s)\right]\right\}$. 
This further suggests us to consider the following two functions:

$$
\Delta_{s, t}(x)= \begin{cases}{\left[\frac{\psi(x+t)-\psi(x+s)}{t-s}\right]^{2}+\frac{\psi^{\prime}(x+t)-\psi^{\prime}(x+s)}{t-s},} & s \neq t \\ {\left[\psi^{\prime}(x+s)\right]^{2}+\psi^{\prime \prime}(x+s),} & s=t\end{cases}
$$

and

$$
\Theta_{s, t}(x)=[\psi(x+t)-\psi(x+s)]^{2}+(t-s)\left[\psi^{\prime}(x+t)-\psi^{\prime}(x+s)\right]
$$

on $x \in(-\alpha, \infty)$ for real numbers $s$ and $t$ and $\alpha=\min \{s, t\}$.

In $[3$, p. 208] and [7, Lemma 1.1], the inequality

$$
\Delta_{0,0}(x)=\left[\psi^{\prime}(x)\right]^{2}+\psi^{\prime \prime}(x)>0
$$

on $(0, \infty)$ was verified. In $[4,7]$, this inequality was applied to provide some symmetrical upper and lower bounds for $\Gamma(x)$ in terms of $\psi(x)$ as follows:

(13) $\exp \left\{\alpha\left[e^{\psi(x)}(\psi(x)-1)+1\right]\right\} \leq \frac{\Gamma(x)}{\Gamma\left(x^{*}\right)} \leq \exp \left\{\beta\left[e^{\psi(x)}(\psi(x)-1)+1\right]\right\}$,

where $x^{*}=1.4616 \cdots$ denotes the only positive zero of $\psi(x)$, and $\alpha$ and $\beta$ are real constants.

The first aim of this paper is to present the completely monotonic property of the functions $\Delta_{s, t}(x)$ and $\Theta_{s, t}(x)$ on $(-\alpha, \infty)$, which implies the positivity of the function $\Delta_{0,0}(x)$ defined by $(12)$.

Theorem 1. The functions $\Delta_{s, t}(x)$ for $|t-s|<1$ and $-\Delta_{s, t}(x)$ for $|t-s|>1$ are completely monotonic on $x \in(-\alpha, \infty)$. So are the functions $\Theta_{s, t}(x)$ for $|t-s|<1$ and $-\Theta_{s, t}(x)$ for $|t-s|>1$ on $x \in(-\alpha, \infty)$.

The second aim of this paper is, by using Theorem 1, to generalize the inequality (13) to a monotonic property as follows.

Theorem 2. For real numbers $s$ and $t, \alpha=\min \{s, t\}$ and $c \in(-\alpha, \infty)$, let

$$
g_{s, t}(x)= \begin{cases}\frac{1}{t-s} \int_{c}^{x} \ln \left[\frac{\Gamma(u+t)}{\Gamma(u+s)} \frac{\Gamma(c+s)}{\Gamma(c+t)}\right] \mathrm{d} u, & s \neq t \\ \int_{c}^{x}[\psi(u+s)-\psi(c+s)] \mathrm{d} u, & s=t\end{cases}
$$

on $x \in(-\alpha, \infty)$. Then the function

$$
f_{s, t}(x)= \begin{cases}\frac{g_{s, t}(x)}{\left[g_{s, t}^{\prime}(x)-1\right] \exp \left[g_{s, t}^{\prime}(x)\right]+1}, & x \neq c \\ \frac{1}{g_{s, t}^{\prime \prime}(c)}, & x=c\end{cases}
$$

on $x \in(-\alpha, \infty)$ is decreasing for $|t-s|<1$ and increasing for $|t-s|>1$.

The third aim of this paper is, by making use of Theorem 1, to provide an alternative proof for the monotonicity and convexity of the function $z_{s, t}(x)$, which is quoted as follows. 
Theorem 3. The function $z_{s, t}(x)$ on $(-\alpha, \infty)$ is either convex and decreasing for $|t-s|<1$ or concave and increasing for $|t-s|>1$.

It is well-known [46] that Wallis cosine or sine formula is

$$
\begin{aligned}
\int_{0}^{\pi / 2} \sin ^{n} x \mathrm{~d} x & =\int_{0}^{\pi / 2} \cos ^{n} x \mathrm{~d} x \\
& =\frac{\sqrt{\pi} \Gamma((n+1) / 2)}{n \Gamma(n / 2)}= \begin{cases}\frac{\pi}{2} \cdot \frac{(n-1) ! !}{n ! !} & \text { for } n \text { even, } \\
\frac{(n-1) ! !}{n ! !} & \text { for } n \text { odd }\end{cases}
\end{aligned}
$$

where $n$ !! denotes the double factorial. It has been estimated by many mathematicians and a lot of inequalities were established in, for example, [10, 12, 13, $14,15,23,25,37,45,49]$ and related references therein.

The fourth aim of this paper is, by utilizing Theorem 3, to prove two sharp double inequalities relating to Wallis cosine or sine formula (16) and to bound the probability integral or error function as follows.

Theorem 4. For $n \in \mathbb{N}$,

$$
\begin{aligned}
& \frac{1}{\sqrt{\pi(n+4 / \pi-1)}} \leq \frac{(2 n-1) ! !}{(2 n) ! !}<\frac{1}{\sqrt{\pi(n+1 / 4)}} \\
& \frac{\sqrt{\pi}}{2 \sqrt{n+9 \pi / 16-1}} \leq \frac{(2 n) ! !}{(2 n+1) ! !}<\frac{\sqrt{\pi}}{2 \sqrt{n+3 / 4}}
\end{aligned}
$$

and

$$
\frac{\sqrt{\pi}}{\sqrt{1+(9 \pi / 16-1) / n}} \leq \int_{-\sqrt{n}}^{\sqrt{n}} e^{-x^{2}} \mathrm{~d} x<\frac{\sqrt{\pi}}{\sqrt{1-3 /(4 n)}} .
$$

In particular, taking $n \rightarrow \infty$ in (19) leads to

$$
\int_{-\infty}^{\infty} e^{-x^{2}} \mathrm{~d} x=\sqrt{\pi}
$$

The constants $\frac{4}{\pi}-1$ and $\frac{1}{4}$ in (17) and the constants $\frac{9 \pi}{16}-1$ and $\frac{3}{4}$ in (18) are the best possible.

The final aim of this paper is, by employing Theorem 3, to recover a double inequality for ratio of the volumes of the unit balls in $\mathbb{R}^{n-1}$ and $\mathbb{R}^{n}$ respectively as follows.

Theorem 5 ([2, Theorem 2]). For $n \in \mathbb{N}$, the inequality

$$
\sqrt{\frac{n+A}{2 \pi}}<\frac{\Omega_{n-1}}{\Omega_{n}} \leq \sqrt{\frac{n+B}{2 \pi}}
$$

holds if and only if $A \leq \frac{1}{2}$ and $B \geq \frac{\pi}{2}-1$, where $\Omega_{n}=\frac{\pi^{n / 2}}{\Gamma(1+n / 2)}$ is the volume of the unit ball on $\mathbb{R}^{n}$. 


\section{Lemmas}

In order to prove our theorems, the following lemmas are necessary.

Lemma 1. Let $f(x)$ be defined in an infinite interval $I$. If

$$
\lim _{x \rightarrow \infty} f(x)=\delta \quad \text { and } \quad f(x)-f(x+\varepsilon)>0
$$

for some given $\varepsilon>0$, then $f(x)>\delta$ on $I$.

Proof. By induction, for any $x \in I$, we have

$$
f(x)>f(x+\varepsilon)>f(x+2 \varepsilon)>\cdots>f(x+k \varepsilon) \rightarrow \delta
$$

as $k \rightarrow \infty$. The proof of Lemma 1 is complete.

Lemma 2 ([1]). For any positive integer $n \in \mathbb{N}$ and $x>0$,

As $x \rightarrow \infty$,

$$
\begin{gathered}
\psi(x)=\ln x+\int_{0}^{\infty}\left[\frac{1}{u}-\frac{1}{1-e^{-u}}\right] e^{-x u} \mathrm{~d} u, \\
\psi^{(n)}(x)=(-1)^{n+1} \int_{0}^{\infty} \frac{u^{n}}{1-e^{-u}} e^{-x u} \mathrm{~d} u, \\
\psi^{(n-1)}(x+1)=\psi^{(n-1)}(x)+\frac{(-1)^{n-1}(n-1) !}{x^{n}}, \\
\ln x-\frac{1}{x}<\psi(x)<\ln x-\frac{1}{2 x} .
\end{gathered}
$$

$$
\psi^{\prime}(x) \sim \frac{1}{x}+\frac{1}{2 x^{2}}+\cdots .
$$

Lemma 3 ([34]). For $s>r>0$,

$$
\exp [(s-r) \psi(s)]>\frac{\Gamma(s)}{\Gamma(r)}>\exp [(s-r) \psi(r)] .
$$

Lemma 4 ([48]). A product of finite completely monotonic functions is also completely monotonic.

\section{Proofs of theorems}

Now we are in a position to prove our theorems.

Proof of Theorem 1. Direct computation and utilization of (24) gives

$$
\begin{aligned}
& \Theta_{s, t}(x)-\Theta_{s, t}(x+1) \\
= & \{[\psi(x+t)+\psi(x+t+1)] \\
& -[\psi(x+s)+\psi(x+s+1)]\}\{[\psi(x+t)-\psi(x+t+1)] \\
& -[\psi(x+s)-\psi(x+s+1)]\}+(t-s)\left\{\left[\psi^{\prime}(x+t)-\psi^{\prime}(x+t+1)\right]\right. \\
& \left.-\left[\psi^{\prime}(x+s)-\psi^{\prime}(x+s+1)\right]\right\}
\end{aligned}
$$




$$
\begin{aligned}
& =\left\{\frac{[\psi(x+t+1)+\psi(x+t)]-[\psi(x+s+1)+\psi(x+s)]}{t-s}\right. \\
& \left.-\frac{2 x+s+t}{(x+s)(x+t)}\right\} \frac{(t-s)^{2}}{(x+s)(x+t)} \\
& \triangleq \Lambda_{s, t}(x) \frac{(t-s)^{2}}{(x+s)(x+t)}
\end{aligned}
$$

and

$$
\begin{aligned}
\Lambda_{s, t}(x)-\Lambda_{s, t}(x+1)= & \frac{1}{t-s}\left(\frac{1}{x+s}+\frac{1}{x+s+1}-\frac{1}{x+t}-\frac{1}{x+t+1}\right) \\
& -\frac{2 x^{2}+2(s+t+1) x+s^{2}+t^{2}+s+t}{(x+s)(x+s+1)(x+t)(x+t+1)} \\
= & \frac{1-(s-t)^{2}}{(x+s)(x+s+1)(x+t)(x+t+1)} .
\end{aligned}
$$

By $(22)$ and (23), it is easy to see that $\lim _{x \rightarrow \infty} \Lambda_{s, t}^{(i)}(x)=0$ for any nonnegative integer $i$. By Lemma 4, it is derived that the function

$$
\frac{\Lambda_{s, t}(x)-\Lambda_{s, t}(x+1)}{1-(s-t)^{2}}
$$

is completely monotonic, that is,

$$
(-1)^{i} \frac{\left[\Lambda_{s, t}(x)-\Lambda_{s, t}(x+1)\right]^{(i)}}{1-(s-t)^{2}}=\frac{(-1)^{i} \Lambda_{s, t}^{(i)}(x)-(-1)^{i} \Lambda_{s, t}^{(i)}(x+1)}{1-(s-t)^{2}} \geq 0
$$

on $(-\alpha, \infty)$. In the light of Lemma 1 , it is deduced that $\frac{(-1)^{i} \Lambda_{s, t}^{(i)}(x)}{1-(s-t)^{2}} \geq 0$, which means that the function $\frac{\Lambda_{s, t}(x)}{1-(s-t)^{2}}$ is completely monotonic on $(-\alpha, \infty)$.

Since the function $\frac{(t-s)^{2}}{(x+s)(x+t)}$ is completely monotonic, then by considering (28) and Lemma 4 it follows that the function

$$
\frac{\Theta_{s, t}(x)-\Theta_{s, t}(x+1)}{1-(s-t)^{2}}
$$

is completely monotonic on $(-\alpha, \infty)$, which is equivalent to

$$
(-1)^{k}\left[\frac{\Theta_{s, t}(x)-\Theta_{s, t}(x+1)}{1-(s-t)^{2}}\right]^{(k)}=\frac{(-1)^{k} \Theta_{s, t}^{(k)}(x)-(-1)^{k} \Theta_{s, t}^{(k)}(x+1)}{1-(s-t)^{2}} \geq 0
$$

for nonnegative integer $k$. Further, from $\lim _{x \rightarrow \infty} \Theta_{s, t}^{(k)}(x)=0$ for nonnegative integer $k$, which can be deduced by utilizing (22) and (23), and Lemma 1, it is concluded that $\frac{(-1)^{k} \Theta_{s, t}^{(k)}(x)}{1-(s-t)^{2}} \geq 0$ for any nonnegative integer $k$. This implies $(-1)^{k} \Theta_{s, t}^{(k)}(x) \gtreqless 0$ if and only if $|t-s| \lessgtr 1$. Therefore, the functions $\Theta_{s, t}(x)$ for $|t-s|<1$ and $-\Theta_{s, t}(x)$ for $|t-s|>1$ are completely monotonic on $(-\alpha, \infty)$. 
Since $\Theta_{s, t}(x)=(t-s)^{2} \Delta_{s, t}(x)$, the function $\Delta_{s, t}(x)$ has the same monotonicity property as $\Theta_{s, t}(x)$ on $(-\alpha, \infty)$. The proof of Theorem 1 is complete.

Proof of Theorem 2. Straightforward computation yields

$$
g_{s, t}^{\prime}(x)= \begin{cases}\frac{1}{t-s} \ln \left[\frac{\Gamma(x+t)}{\Gamma(x+s)} \frac{\Gamma(c+s)}{\Gamma(c+t)}\right], & s \neq t \\ \psi(x+s)-\psi(c+s), & s=t .\end{cases}
$$

It is clear that $g_{s, t}(c)=g_{s, t}^{\prime}(c)=0$ and

$$
g_{s, t}^{\prime \prime}(x)= \begin{cases}\frac{\psi(x+t)-\psi(x+s)}{t-s}>0, & s \neq t \\ \psi^{\prime}(x+s)>0, & s=t .\end{cases}
$$

This leads to $(x-c) g_{s, t}^{\prime}(x)>0$ and $g_{s, t}(x)>0$ for $x \neq c$.

Differentiation yields

$$
f_{s, t}^{\prime}(x)=\frac{g_{s, t}^{\prime}(x) h_{s, t}(x)}{\left\{\left[g_{s, t}^{\prime}(x)-1\right] \exp \left[g_{s, t}^{\prime}(x)\right]+1\right\}^{2}},
$$

where

$$
h_{s, t}(x)=\left[g_{s, t}^{\prime}(x)-g_{s, t}(x) g_{s, t}^{\prime \prime}(x)-1\right] \exp \left[g_{s, t}^{\prime}(x)\right]+1 .
$$

Since $h_{s, t}(c)=0,\left[g_{s, t}^{\prime \prime}(x)\right]^{2}+g_{s, t}^{\prime \prime \prime}(x)>0$ by Theorem 1 , and

$$
h_{s, t}^{\prime}(x)=-g_{s, t}(x)\left\{\left[g_{s, t}^{\prime \prime}(x)+g_{s, t}^{\prime \prime \prime}(x)\right]^{2}\right\} \exp \left[g_{s, t}^{\prime}(x)\right] \lessgtr 0
$$

for $|t-s| \lessgtr 1$ and $x \neq c$, we obtain $(x-c) h_{s, t}(x) \lessgtr 0$ and hence $f_{s, t}^{\prime}(x) \lessgtr 0$ for $|t-s| \lessgtr 1$ and $x \neq c$.

Proof of Theorem 3. It is clear from (8) and (9) that

$$
z_{s, t}^{\prime \prime}(x)=\left[z_{s, t}(x)+x\right] \Delta_{s, t}(x)=\frac{z_{s, t}(x)+x}{(t-s)^{2}} \Theta_{s, t}(x)
$$

for $t \neq s$. By Theorem 1 , it is easy to see that $\Theta_{s, t}(x) \gtreqless 0$ and $\Delta_{s, t}(x) \gtreqless 0$ on $(-\alpha, \infty)$ if and only if $|t-s| \lessgtr 1$. Then $z_{s, t}^{\prime \prime}(x) \gtreqless 0$ for $|t-s| \lessgtr 1$ follows from formula (30). The convexity and concavity of the function $z_{s, t}(x)$ is proved.

The inequality $(27)$ is equivalent to

$$
\max \left\{e^{\psi(s)}, e^{\psi(r)}\right\}>\left[\frac{\Gamma(s)}{\Gamma(r)}\right]^{1 /(s-r)}>\min \left\{e^{\psi(s)}, e^{\psi(r)}\right\}
$$


for any positive numbers $s>0$ and $t>0$. This implies

$$
\begin{aligned}
z_{s, t}^{\prime}(x) & =\left[\frac{\Gamma(x+t)}{\Gamma(x+s)}\right]^{1 /(t-s)} \frac{\psi(x+t)-\psi(x+s)}{t-s}-1 \\
& <e^{\psi(x+t)} \frac{\psi(x+t)-\psi(x+s)}{t-s}-1 \\
& =e^{\psi(x+t)} \psi^{\prime}(x+\xi)-1 \\
& <\psi^{\prime}(x+t) e^{\psi(x+t)}-1
\end{aligned}
$$

and

$$
\begin{aligned}
z_{s, t}^{\prime}(x) & >e^{\psi(x+s)} \frac{\psi(x+t)-\psi(x+s)}{t-s}-1 \\
& =e^{\psi(x+s)} \psi^{\prime}(x+\xi)-1 \\
& >\psi^{\prime}(x+s) e^{\psi(x+s)}-1
\end{aligned}
$$

if assuming $t>s>0$ without loss of generality, where $\xi \in(s, t)$.

By the inequality (25), we obtain

$$
x \psi^{\prime}(x) e^{-1 / x}<\psi^{\prime}(x) e^{\psi(x)}<x \psi^{\prime}(x) e^{-1 / 2 x}
$$

for $x>0$. Using the asymptotic representation (26) yields

$$
\lim _{x \rightarrow \infty}\left[x \psi^{\prime}(x) e^{-1 / x}\right]=1 \quad \text { and } \quad \lim _{x \rightarrow \infty}\left[x \psi^{\prime}(x) e^{-1 / 2 x}\right]=1 .
$$

Hence,

$$
\lim _{x \rightarrow \infty}\left[\psi^{\prime}(x) e^{\psi(x)}\right]=1 .
$$

Combining (35) with (31) and (32) leads to

$$
\begin{aligned}
\lim _{x \rightarrow \infty} z_{s, t}^{\prime}(x) & \leq \lim _{x \rightarrow \infty}\left[\psi^{\prime}(x+t) e^{\psi(x+t)}\right]-1 \\
& =\lim _{x+t \rightarrow \infty}\left[\psi^{\prime}(x+t) e^{\psi(x+t)}\right]-1 \\
& =0
\end{aligned}
$$

and

$$
\begin{aligned}
\lim _{x \rightarrow \infty} z_{s, t}^{\prime}(x) & \geq \lim _{x \rightarrow \infty}\left[\psi^{\prime}(x+s) e^{\psi(x+s)}\right]-1 \\
& =\lim _{x+s \rightarrow \infty}\left[\psi^{\prime}(x+s) e^{\psi(x+s)}\right]-1 \\
& =0 .
\end{aligned}
$$

Thus, it is concluded that $\lim _{x \rightarrow \infty} z_{s, t}^{\prime}(x)=0$.

Since $z_{s, t}^{\prime \prime}(x) \gtreqless 0$ on $x \in(-\alpha, \infty)$ for $|t-s| \lessgtr 1$, then the function $z_{s, t}^{\prime}(x)$ is increasing/decreasing on $x \in(-\alpha, \infty)$ for $|t-s| \lessgtr 1$. Thus, it follows that $z_{s, t}^{\prime}(x) \lesseqgtr 0$ and $z_{s, t}(x)$ is decreasing/increasing in $x \in(-\alpha, \infty)$ for $|t-s| \lessgtr 1$. The monotonicity of the function $z_{s, t}(x)$ is proved. 
The second proof of convexity of $z_{s, t}(x)$. It is sufficient to show the function

$$
\Phi_{s, t}(x)= \begin{cases}\frac{\psi(x+s)-\psi(x+t)}{s-t}\left[\frac{\Gamma(x+s)}{\Gamma(x+t)}\right]^{1 /(s-t)}, & s \neq t \\ \psi^{\prime}(x+s) e^{\psi(x+s)}, & s=t\end{cases}
$$

on $(-\alpha, \infty)$ is increasing for $|t-s|<1$ and decreasing for $|t-s|>1$.

Straightforward calculation yields

$$
\ln \Phi_{s, t}(x)= \begin{cases}\ln \frac{\psi(x+s)-\psi(x+t)}{s-t}+\frac{\ln \Gamma(x+s)-\ln \Gamma(x+t)}{s-t}, & s \neq t \\ \ln \psi^{\prime}(x+s)+\psi(x+s), & s=t\end{cases}
$$

and

$$
\begin{aligned}
{\left[\ln \Phi_{s, t}(x)\right]^{\prime} } & = \begin{cases}\frac{\psi^{\prime}(x+s)-\psi^{\prime}(x+t)}{\psi(x+s)-\psi(x+t)}+\frac{\psi(x+s)-\psi(x+t)}{s-t}, & s \neq t \\
\frac{\psi^{\prime \prime}(x+s)}{\psi^{\prime}(x+s)}+\psi^{\prime}(x+s), & s=t\end{cases} \\
& = \begin{cases}\frac{s-t}{\psi(x+s)+\psi(x+t)} \Delta_{s, t}(x), & s \neq t \\
\frac{1}{\psi^{\prime}(x+s)} \Delta_{s, s}(x), & s=t .\end{cases}
\end{aligned}
$$

In virtue of Theorem 1 , it is concluded that

$$
\left[\ln \Phi_{s, t}(x)\right]^{\prime} \begin{cases}>0, & \text { if }|t-s|<1 \\ <0, & \text { if }|t-s|>1\end{cases}
$$

The second proof of convexity of $z_{s, t}(x)$ is complete.

Proof of Theorem 4. Let the sequence $\theta_{1}(n)$ be defined by

$$
\frac{(2 n-1) ! !}{(2 n) ! !}=\frac{1}{\sqrt{\pi\left(n+\theta_{1}(n)\right)}}
$$

for $n \in \mathbb{N}$. In order to obtain the inequality (17), it is sufficient to show

$$
\frac{1}{4}<\theta_{1}(n) \leq \frac{4}{\pi}-1
$$

for all $n \in \mathbb{N}$. Indeed, formula (16) and Theorem 3 implies

$$
\theta_{1}(x)=\left[\frac{\Gamma(x+1)}{\Gamma(x+1 / 2)}\right]^{2}-x=z_{1 / 2,1}(x)
$$

is convex and decreasing on $\left(-\frac{1}{2}, \infty\right)$, and then the sharp double inequality (38) can be deduced by observing that $\theta_{1}(1)=\frac{4}{\pi}-1$ and $\lim _{x \rightarrow \infty} \theta_{1}(x)=\frac{1}{4}$.

Let the sequence $\theta_{2}(n)$ be defined by

$$
\frac{(2 n) ! !}{(2 n+1) ! !}=\frac{\sqrt{\pi}}{2 \sqrt{n+\theta_{2}(n)}}
$$


for $n \in \mathbb{N}$. In order to obtain the inequality (18), it is sufficient to show

$$
\frac{3}{4}<\theta_{2}(n) \leq \frac{9 \pi}{16}-1
$$

for all $n \in \mathbb{N}$. Indeed, formula (16) and Theorem 3 implies

$$
\theta_{2}(x)=\left[\frac{\Gamma(x+3 / 2)}{\Gamma(x+1)}\right]^{2}-x=z_{1,3 / 2}(x)
$$

is convex and decreasing on $(-1, \infty)$, and then the sharp double inequality (41) can be deduced by observing that $\theta_{2}(1)=\frac{9 \pi}{16}-1$ and $\lim _{x \rightarrow \infty} \theta_{2}(x)=\frac{3}{4}$.

The rest is the same as the proof of [10, Theorem 1.2].

Proof of Theorem 5. The inequality (21) can be rearranged as

$$
\frac{A}{2}<z_{1,1 / 2}\left(\frac{n}{2}\right) \leq \frac{B}{2}
$$

for $n \in \mathbb{N}$. Since $z_{1,1 / 2}(x)$ is decreasing on $\left(-\frac{1}{2}, 1\right)$ by Theorem 3 , considering $z_{1,1 / 2}\left(\frac{1}{2}\right)=\frac{\pi}{4}-\frac{1}{2}$ and $\lim _{x \rightarrow \infty} z_{1,1 / 2}(x)=\frac{1}{4}$, then the inequality (43) is concluded.

\section{Remarks}

In this section, we would like to give several remarks about our theorems.

Remark 1. If taking $c=x^{*}$, then the case $s=t$ in Theorem 2 becomes $[4$, Theorem 4.3]: For $0<a<b \leq \infty$ and $x \in(a, b)$, the inequality (13) holds with the best possible constant factors

$$
\alpha=\left\{\begin{array}{ll}
Q(b), & b<\infty \\
1, & b=\infty
\end{array} \text { and } \beta=Q(a),\right.
$$

where

$$
Q(x)= \begin{cases}\frac{\ln \Gamma(x)-\ln \Gamma\left(x^{*}\right)}{e^{\psi(x)}[\psi(x)-1]+1}, & x \neq x^{*} ; \\ \frac{1}{\psi^{\prime}\left(x^{*}\right)}, & x=x^{*}\end{cases}
$$

Remark 2. Theorem 3 has been proved in $[11,17,21,27,40,44]$ once again. For more information on this topic, please refer to the expository and survey articles [32, 33] and plenty of references therein.

Remark 3. In $[6,7,17]$, the inequalities

$$
\psi^{\prime}(x) e^{\psi(x)}<1, \quad x>0
$$

and (12) were proved and used to construct many inequalities for bounding the gamma function $\Gamma(x)$, the psi function $\psi$ and the tri-gamma function $\psi^{\prime}(x)$ such as (13). 
In [17], as a corollary of Theorem 3, the following inequality was deduced:

$$
\left[\frac{\Gamma(x+t)}{\Gamma(x+s)}\right]^{1 /(t-s)}<\frac{t-s}{\psi(x+t)-\psi(x+s)}
$$

holds for $0<|t-s|<1$ and with reversed sign if $|t-s|>1$.

The second proof of convexity of $z_{s, t}(x)$ implies that inequalities (12), (46) and (47) are equivalent to each other.

Remark 4. Recall [5, 39, 42] that a positive function $f$ is called logarithmically completely monotonic on an interval $I$ if $f$ has derivatives of all orders on $I$ and its logarithm $\ln f$ satisfies

$$
(-1)^{k}[\ln f(x)]^{(k)} \geq 0
$$

for all $k \in \mathbb{N}$ on $I$. It was proved explicitly in $[8,20,36,39]$ by different approaches that any logarithmically completely monotonic function must be completely monotonic, but not conversely. It was pointed out in $[8$, Theorem 1.1] and [19, 43] that the logarithmically completely monotonic functions on $[0, \infty)$ are those completely monotonic functions on $[0, \infty)$ for which the representing measure $\mu$ in (2) is infinitely divisible in the convolution sense: For each $n \in \mathbb{N}$ there exists a positive measure $\nu$ on $[0, \infty)$ with $n$-th convolution power equal to $\mu$. By the way, the preprints $[39,42]$ have been extended, revised, and formally published in $[22,36,43]$ respectively.

We conjecture that the function $\Phi_{s, t}(x)$, defined by $(36)$, for $|t-s|>1$ and its reciprocal for $|t-s|<1$ are logarithmically completely monotonic on $x \in(-\alpha, \infty)$. This modifies a part of [44, p. 434, Open Problem 2].

\section{References}

[1] M. Abramowitz and I. A. Stegun, Handbook of Mathematical Functions with Formulas, Graphs, and Mathematical Tables, National Bureau of Standards, Applied Mathematics Series 55, 4th printing, with corrections, Washington, 1965.

[2] H. Alzer, Inequalities for the volume of the unit ball in $\mathbb{R}^{n}$, J. Math. Anal. Appl. 252 (2000), no. 1, 353-363.

[3] Sharp inequalities for the digamma and polygamma functions, Forum Math. 16 (2004), no. 2, 181-221.

[4] H. Alzer and A. Z. Grinshpan, Inequalities for the gamma and q-gamma functions, J. Approx. Theory 144 (2007), no. 1, 67-83.

[5] R. D. Atanassov and U. V. Tsoukrovski, Some properties of a class of logarithmically completely monotonic functions, C. R. Acad. Bulgare Sci. 41 (1988), no. 2, 21-23.

[6] N. Batir, An interesting double inequality for Euler's gamma function, J. Inequal. Pure Appl. Math. 5 (2004), no. 4, Article 97, 3 pp.; Available online at http://www.emis.de/ journals /JIPAM/article452.html?sid=452.

[7] Some new inequalities for gamma and polygamma functions, J. Inequal. Pure Appl. Math. 6 (2005), no. 4, Article 103, 9 pp.; Available online at http://www.emis.de/ journals/JIPAM/article577.html?sid $=577$.

[8] C. Berg, Integral representation of some functions related to the gamma function, Mediterr. J. Math. 1 (2004), no. 4, 433-439.

[9] J. Bustoz and M. E. H. Ismail, On gamma function inequalities, Math. Comp. 47 (1986), no. $176,659-667$. 
[10] J. Cao, D.-W. Niu, and F. Qi, A Wallis type inequality and a double inequality for probability integral, Aust. J. Math. Anal. Appl. 4 (2007), no. 1, Art. 3, 6 pp.; Available online at http://ajmaa.org/cgi-bin/paper.pl?string $=\mathrm{v} 4 \mathrm{n} 1 / \mathrm{V} 4 \mathrm{I} 1 \mathrm{P} 3$. tex.

[11] Ch.-P. Chen, Monotonicity and convexity for the gamma function, J. Inequal. Pure Appl. Math. 6 (2005), no. 4, Article 100, 6 pp.; Available online at http://www.emis.de/ journals $/$ JIPAM/article $457 . h t m l ?$ sid $=457$.

[12] Ch.-P. Chen and F. Qi, Best upper and lower bounds in Wallis' inequality, J. Indones. Math. Soc. 11 (2005), no. 2, 137-141.

[13] Completely monotonic function associated with the gamma functions and proof of Wallis' inequality, Tamkang J. Math. 36 (2005), no. 4, 303-307.

[14] $2,397-401$.

[15]

[16] J. Dutka, On some gamma function inequalities, SIAM J. Math. Anal. 16 (1985), no. $1,180-185$.

[17] N. Elezović, C. Giordano and J. Pečarić, The best bounds in Gautschi's inequality, Math. Inequal. Appl. 3 (2000), no. 2, 239-252.

[18] W. Gautschi, Some elementary inequalities relating to the gamma and incomplete gamma function, J. Math. Phys. 38 (1959/60), 77-81.

[19] A. Z. Grinshpan and M. E. H. Ismail, Completely monotonic functions involving the gamma and q-gamma functions, Proc. Amer. Math. Soc. 134 (2006), no. 4, 1153-1160.

[20] B.-N. Guo and F. Qi, A property of logarithmically absolutely monotonic functions and the logarithmically complete monotonicity of a power-exponential function, Politehn. Univ. Bucharest Sci. Bull. Ser. A Appl. Math. Phys. 72 (2010), no. 2, 21-29.

[21] _ An alternative proof of Elezović-Giordano-Pečarić's theorem, Math. Inequal. Appl. 14 (2011), no. 1, in press.

[22] _ Some logarithmically completely monotonic functions related to the gamma function, J. Korean Math. Soc. 47 (2010), no. 6, 1283-1297; Available online at http://dx.doi.org/10.4134/JKMS.2010.47.6.1283.

[23] D. K. Kazarinoff, On Wallis' formula, Edinburgh Math. Notes 1956 (1956), no. 40, 19-21.

[24] D. Kershaw, Some extensions of W. Gautschi's inequalities for the gamma function, Math. Comp. 41 (1983), no. 164, 607-611.

[25] S. Koumandos, Remarks on a paper by Chao-Ping Chen and Feng Qi, Proc. Amer. Math. Soc. 134 (2006), no. 5, 1365-1367 .

[26] A. Laforgia, Further inequalities for the gamma function, Math. Comp. 42 (1984), no. $166,597-600$.

[27] I. Lazarević and A. Lupaş, Functional equations for Wallis and gamma functions, Univ. Beograd. Publ. Elektrotehn. Fak. Ser. Mat. Fiz. No. 461-497 (1974), 245-251.

[28] D. S. Mitrinović, J. E. Pečarić, and A. M. Fink, Classical and New Inequalities in Analysis, Kluwer Academic Publishers, 1993.

[29] F. Qi, A class of logarithmically completely monotonic functions and application to the best bounds in the second Gautschi-Kershaw's inequality, J. Comput. Appl. Math. 224 (2009), no. 2, 538-543; Available online at http://dx.doi.org/10.1016/j.cam.2008.05.030.

[30] $\ldots$ A class of logarithmically completely monotonic functions and the best bounds in the first Kershaw's double inequality, J. Comput. Appl. Math. 206 (2007), no. 2, 1007-1014; Available online at http://dx.doi.org/10.1016/j.cam.2006.09.005.

[31] _ _ A completely monotonic function involving the divided difference of the psi function and an equivalent inequality involving sums, ANZIAM J. 48 (2007), no. 4, 523-532.

[32] _ Bounds for the ratio of two gamma functions, J. Inequal. Appl. 2010 (2010), Art. ID 493058, 84 pp.; Available online at http://dx.doi.org/10.1155/2010/493058. 
[33] B__ Bounds for the ratio of two gamma functions-From Wendel's limit to ElezovićGiordano-Pečarić's theorem, Available online at http://arxiv.org/abs/0902.2514.

[34] _ Monotonicity results and inequalities for the gamma and incomplete gamma functions, Math. Inequal. Appl. 5 (2002), no. 1, 61-67.

[35] _ Three classes of logarithmically completely monotonic functions involving gamma and psi functions, Integral Transforms Spec. Funct. 18 (2007), no. 7-8, 503509; Available online at http://dx.doi.org/10.1080/10652460701358976.

[36] F. Qi and Ch.-P. Chen, A complete monotonicity property of the gamma function, J. Math. Anal. Appl. 296 (2004), no. 2, 603-607; Available online at http://dx.doi.org/ $10.1080 / 10652460701318418$.

[37] F. Qi, L.-H. Cui, and S.-L. Xu, Some inequalities constructed by Tchebysheff's integral inequality, Math. Inequal. Appl. 2 (1999), no. 4, 517-528.

[38] F. Qi and B.-N. Guo, A class of logarithmically completely monotonic functions and the best bounds in the second Kershaw's double inequality, J. Comput. Appl. Math. 212 (2008), no. 2, 444-456; Available online at http://dx.doi.org/10.1016/j.cam.2006.12.022.

[39] - Complete monotonicities of functions involving the gamma and digamma functions, RGMIA Res. Rep. Coll. 7 (2004), no. 1, Art. 8, 63-72; Available online at http://rgmia.org/v7n1.php.

[40] - Completely monotonic functions involving divided differences of the di- and tri-gamma functions and some applications, Commun. Pure Appl. Anal. 8 (2009), no. 6, 1975-1989; Available online at http://dx.doi.org/10.3934/cpaa.2009.8.1975.

[41] _ Wendel's and Gautschi's inequalities: refinements, extensions, and a class of logarithmically completely monotonic functions, Appl. Math. Comput. 205 (2008), no. 1, 281-290; Available online at http://dx.doi.org/10.1016/j.amc.2008.07.005.

[42] F. Qi, B.-N. Guo, and Ch.-P. Chen, Some completely monotonic functions involving the gamma and polygamma functions, RGMIA Res. Rep. Coll. 7 (2004), no. 1, Art. 5, 31-36; Available online at http://rgmia.org/v7n1.php.

[43] _ Some completely monotonic functions involving the gamma and polygamma functions, J. Aust. Math. Soc. 80 (2006), no. 1, 81-88.

[44] - The best bounds in Gautschi-Kershaw inequalities, Math. Inequal. Appl. 9 (2006), no. 3, 427-436.

[45] G. N. Watson, A note on Gamma functions, Proc. Edinburgh Math. Soc. (2) 11 (1958/1959); Edinburgh Math. Notes No. 42 (misprinted 41) (1959), 7-9.

[46] E. W. Weisstein, Wallis Cosine Formula, From MathWorld-A Wolfram Web Resource; Available online at http://mathworld.wolfram.com/WallisCosineFormula.html.

[47] J. G. Wendel, Note on the gamma function, Amer. Math. Monthly 55 (1948), no. 9, 563-564.

[48] D. V. Widder, The Laplace Transform, Princeton University Press, Princeton, 1946.

[49] Y.-Q. Zhao and Q.-B. Wu, Wallis inequality with a parameter, J. Inequal. Pure Appl. Math. 7 (2006), no. 2, Article 56, 10 pp.; Available online at http://www.emis.de/ journals/JIPAM/article673.html?sid=673.

\section{BAI-Ni GUO}

SCHOOL OF Mathematics AND Informatics

Henan Polytechnic University

Jiaozuo City, Henan Province, 454010, P. R. China

E-mail address: bai.ni.guo@gmail.com, bai.ni.guo@hotmail.com

FENG QI

SCHOOL OF MATHEMATICS AND INFORMATICS

Henan Polytechnic University

Jiaozuo City, Henan Province, 454010, P. R. China

E-mail address: qifeng618@gmail.com, qifeng618@hotmail.com, qifeng618@qq.com 